\title{
Adiabatic excitation of longitudinal bunch shape oscillations
}

\author{
M. Bai, K. A. Brown, W. Fischer, T. Roser, N. Tsoupas, and J. van Zeijts \\ Brookhaven National Laboratory, Upton, New York 11973
}

(Received 10 April 2000; published 26 June 2000)

\begin{abstract}
By modulating the rf voltage at near twice the synchrotron frequency, the longitudinal bunch shape can be modulated. This method can be used to shorten bunches. We show experimentally that the bunch shape can be modulated while preserving the longitudinal emittance when the rf voltage modulation is turned on adiabatically. Experimental measurements will be presented along with theoretical predictions.
\end{abstract}

PACS numbers: 41.85.Ct, 29.27.Bd, 29.27.Fh

\section{INTRODUCTION}

A modulation of the rf voltage can lead to a modulation of the longitudinal bunch shape. This is particularly pronounced when a parametric resonance is excited by modulating the rf voltage at near twice the synchrotron frequency. Furthermore, when the rf voltage modulation amplitude is increased and decreased adiabatically, i.e., over many synchrotron periods, the longitudinal emittance of the beam can be preserved.

This technique has been used at Brookhaven's Alternating Gradient Synchrotron (AGS) to extract shortened proton bunches that could produce shorter muon pulses for the g-2 experiment [1]. Previous studies with rf voltage modulations are reported in Refs. [2-4]. We present a theoretical description of the parametric resonance used and show experimental data.

\section{LONGITUDINAL MOTION WITH MODULATED FOCUSING STRENGTH}

The synchrotron motion can be described using the conjugate phase space coordinates $\left(\phi, \delta=\frac{h \eta}{\nu_{s}} \frac{\Delta p}{p}\right)$ where $\phi$ is the particle phase relative to the synchronous particle, $h$ is the harmonic number, $\eta=\frac{1}{\gamma_{t}^{2}}-\frac{1}{\gamma^{2}}$ is the phase slip fac- tor, $\nu_{s}$ is the synchrotron tune at zero amplitude without modulation, and $p$ is the particle momentum. The synchrotron tune is given by $\nu_{s}=\sqrt{(h|\eta| e V) / 2 \pi \beta^{2} E}$ with $e$ being the elementary charge, $V$ the gap voltage, $\beta$ the relativistic factor, and $E$ the particle energy. The discrete synchrotron equations for a stationary bucket above transition can then be written as [4,5]

$\begin{aligned} \phi_{n+1} & =\phi_{n}+2 \pi \nu_{s} \delta_{n+1}, \\ \delta_{n+1} & =\delta_{n}+2 \pi \nu_{s}\left[1+\epsilon \sin \left(\nu_{m} \theta_{n+1}+\chi\right)\right] \sin \phi_{n},\end{aligned}$

where $\epsilon$ is the amplitude of $\mathrm{rf}$ voltage modulation, $\nu_{m}$ is the modulation tune, $\chi$ is the initial modulation phase, and the orbital angle $\theta$ is a timelike variable. Equations (1) correspond to the Hamiltonian

$$
H=\frac{\nu_{s}}{2} \delta^{2}+\nu_{s}\left[1+\epsilon \sin \left(\nu_{m} \theta+\chi\right)\right][1-\cos \phi] .
$$

For particles within a bucket, the canonical transformation $\phi=\sqrt{2 J} \cos \psi, \delta=-\sqrt{2 J} \sin \psi$ to action-angle variables $(J, \psi)$ can be used, which allows going into a rotating coordinate frame later. The Hamiltonian can now be approximated by [3]

$$
\begin{gathered}
H=\nu_{s} J-\frac{\nu_{s}}{16} J^{2}+\nu_{s} \epsilon \sin \left(\nu_{m} \theta\right)\left[1-J_{0}(\sqrt{2 J})\right]+\sum_{k=1}^{\infty}\left(\Delta H_{2 k}^{(+)}+\Delta H_{2 k}^{(-)}\right) \\
\text {with } \Delta H_{2 k}^{( \pm)}=\nu_{s} \epsilon(-1)^{k+1} J_{2 k}(\sqrt{2 J}) \sin \left(\nu_{m} \theta \pm 2 k \psi\right) .
\end{gathered}
$$

The $J_{n}$ are Bessel functions of the order $n$ and we have set $\chi=0$ for convenience. If the modulation tune is only slightly different from twice the synchrotron tune, the term $\Delta H_{2}^{(-)}$in Eq. (3) is only slowly changing with time while all other terms $\Delta H_{2 k}^{( \pm)}$are oscillating rapidly. We now go into a coordinate system that rotates around the origin with half the modulation tune by using the canonical transformation $J=\tilde{J}, \tilde{\psi}=\psi-\frac{\nu_{m}}{2} \theta-\frac{3 \pi}{4}$. The new Hamiltonian has terms oscillating at $\nu_{m}, 2 \nu_{m}, \ldots$, which average to zero over time, and the time averaged Hamiltonian in this coordinate system is [3]

$$
\langle\tilde{H}\rangle=\left(\nu_{s}-\frac{\nu_{m}}{2}\right) \tilde{J}-\frac{\nu_{s}}{16} \tilde{J}^{2}+\frac{\nu_{s}}{4} \epsilon \tilde{J} \cos 2 \tilde{\psi} .
$$

Since $J=\tilde{J}$ we use $J$ instead of $\tilde{J}$ in the following.

The investigation of fixed points of the Hamiltonian (4) leads to the following result:

(a) If $\nu_{s}\left(2+\frac{\epsilon}{2}\right)<\nu_{m}$ the origin $J=0$ is the only fixed point, a stable one.

(b) If $\nu_{s}\left(2-\frac{\epsilon}{2}\right)<\nu_{m}<\nu_{s}\left(2+\frac{\epsilon}{2}\right)$ there is one unstable fixed point (UFP) at the origin and two stable fixed points (SFPs) at $J=8\left(1-\frac{\nu_{m}}{2 \nu_{s}}\right)+2 \epsilon$ and $\tilde{\psi}=0, \pi$.

(C) 2000 The American Physical Society

064001-1 
(c) If $\nu_{m}<\nu_{s}\left(2-\frac{\epsilon}{2}\right)$ there is a stable fixed point at the origin, two more stable fixed points at $J=8\left(1-\frac{\nu_{m}}{2 \nu_{s}}\right)+$ $2 \epsilon$ and $\tilde{\psi}=0, \pi$, and two unstable fixed points at $J=$ $8\left(1-\frac{\nu_{m}}{2 \nu_{s}}\right)-2 \epsilon$ and $\tilde{\psi}=\frac{\pi}{2}, \frac{3 \pi}{2}$.

We work in the regime (b) where particles are pushed away from the origin and held in two islands. This phenomenon is known as parametric resonance [6].

The three cases are illustrated in Fig. where we used the approximation (4) and the parameters $\nu_{s}=0.0005$ and $\epsilon=0.45$. Figure (a1) shows the situation with $\nu_{m}=0.0018$ above and far away from the resonance interval $\left[\nu_{s}\left(2-\frac{\epsilon}{2}\right), \nu_{s}\left(2+\frac{\epsilon}{2}\right)\right]$. The phase space trajectories are undisturbed. In Fig. (a2) the modulation tune $\nu_{m}=0.0011$ is still above but close to the resonance interval. The trajectories in phase space are distorted. Figure (b) shows the phase space with $\nu_{m}=0.001$ inside the resonance interval. The unstable fixed point at the
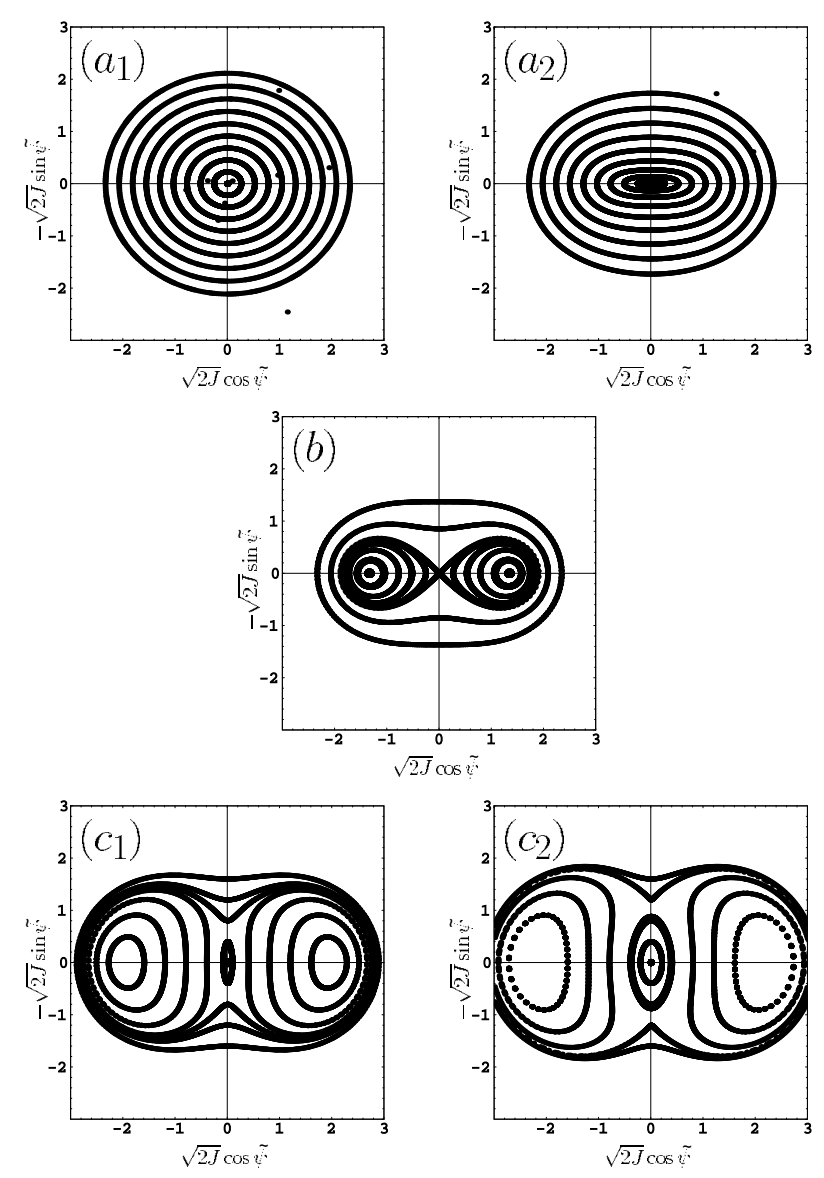

FIG. 1. Particle trajectories in a surface of a section of the Hamiltonian (4) with the parameters $\nu_{s}=0.0005$ and $\epsilon=0.45$. In part (a1) the modulation tune $\nu_{m}=0.0018$ is above and far away from the parametric resonance interval, in part (a2) it is still above but with $\nu_{m}=0.0011$ close to this interval. Part (b) shows the situation with $\nu_{m}=0.001$, when the parametric resonance is excited. Parts (c1) and (c2) depict the cases with $\nu_{m}=0.00087$ and $\nu_{m}=0.0008$, respectively, when the modulation tune is below the parametric resonance and either close or far away from that interval. origin and the two stable fixed points are clearly visible. In Fig. (c1) the modulation tune $\nu_{m}=0.00087$ is below but close to the resonance interval. The appearance of a stable fixed point at the origin and two unstable fixed point above and below it becomes visible. Figure (c2) shows the phase space with $\nu_{m}=0.0008$ below and farther away from the resonance interval.

\section{EXPERIMENTAL RESULTS}

We operated Brookhaven's Alternating Gradient Synchrotron with one bunch of a high intensity proton beam at $24 \mathrm{GeV}$, with a harmonic number of 6 and an rms bunch area of $4 \mathrm{eV} \mathrm{s}$. The unmodulated gap voltage seen by the beam was $180 \mathrm{kV}$. These parameters are summarized in Table I.

Figure 2 shows the part of the low level rf system in which the voltage modulation was implemented. A waveform generator generates the voltage $V_{\text {mod }}$ that is added to the gap voltage setting $V_{0}$. The combined signal is the input to the rf high level voltage regulation.

In our experiments we slowly increased the parameter $\epsilon$ in Eq. (1) to ensure adiabaticity. With a ramp-up time of ten synchrotron periods, we obtain for the adiabaticity parameter $\epsilon_{a}$ [7]

$$
\epsilon_{a}=\frac{1}{\omega_{s}^{2}}\left|\frac{d \omega_{s}}{d t}\right|<0.02 .
$$

Figure 3 shows scope signals for one case in this process: the modulated rf voltage, the beam peak detector signal (peak amplitude of the bunch), and the beam current signal. The rf voltage was driven by an amplitude modulated sinusoidal signal generated by a waveform generator while the average of the rf voltage was kept unperturbed. The beam peak detector measures the peak of the beam pulse obtained from a wall current monitor. Assuming the beam current is constant, the narrower the bunch length, the higher the beam peak detector signal.

The average synchrotron frequency in Fig 3 is $85 \mathrm{~Hz}$, and the rf voltage drive amplitude is $\epsilon=0.8$. The envelopes of the rf voltage signal and the beam peak detector

TABLE I. Basic parameters in the AGS experiment.

\begin{tabular}{lccc}
\hline \hline \multicolumn{1}{c}{ Parameter } & Symbol & Unit & Value \\
\hline Species & $\ldots$ & $\ldots$ & $\mathrm{p}$ \\
Energy & $E$ & $\mathrm{GeV}$ & 24 \\
Harmonic number & $h$ & $\ldots$ & 6 \\
Number of bunches & $\ldots$ & $\ldots$ & 1 \\
Particles per bunch & $\ldots$ & $\ldots$ & $5 \times 10^{12}$ \\
rms bunch area & $\epsilon_{s}$ & $\mathrm{eV} \mathrm{s}$ & 4 \\
Slip factor & $\eta$ & $\ldots$ & 0.0153 \\
Gap voltage & $V_{0}$ & $\mathrm{~V}$ & 190 \\
Synchrotron tune & $\nu_{s}$ & $\ldots$ & 85 \\
\hline \hline
\end{tabular}




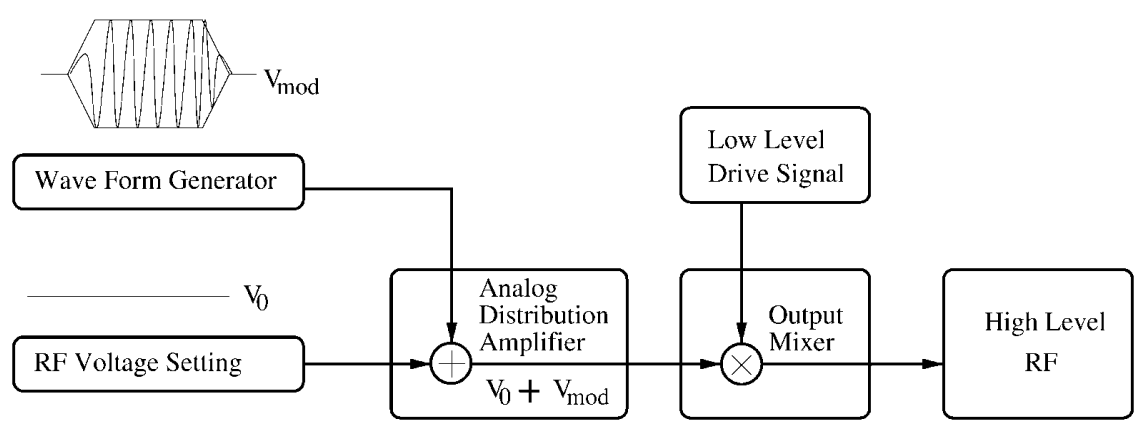

FIG. 2. Overview of the rf voltage modulation system.

signal show that the bunch shape oscillations nicely follow the rf voltage modulation. As the rf voltage modulation is ramped down to zero again, the bunch shape oscillation is also diminished. This indicates that the parametric resonance was excited adiabatically and the process is reversible.

The longitudinal beam profile evolution corresponding to Fig. 3 is shown in Fig. 4 as a mountain range. It clearly shows the bunch shape oscillation when the rf voltage was driven at $185 \mathrm{~Hz}$, slightly above twice the synchrotron oscillation frequency and clearly in regime (b) of the Hamiltonian (4).

Since $\nu_{s}=0.23, \nu_{m}=0.5$, and $\epsilon=0.8$ satisfy

$$
\nu_{s}\left(2-\frac{\epsilon}{2}\right)=0.368<\nu_{m}<\nu_{s}\left(2+\frac{\epsilon}{2}\right)=0.552 \text {, }
$$

two SFPs were developed. The beam was stretched into the two islands around these two SFPs and shaped like a "dumbbell," as shown in the tomographic reconstruction of the bunch in Fig. 5 [8].

The bunch length was measured with different modulation frequencies while the rf voltage drive amplitude was kept fixed at $190 \mathrm{kV}$. Figure 6(a) shows that the minimum

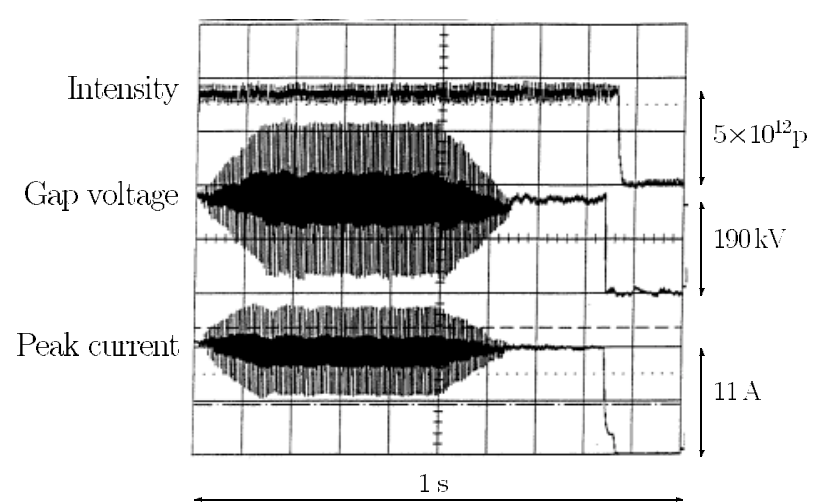

FIG. 3. Typical scope signals with the modulated rf voltage signal, the beam peak detector signal, and the beam current signal. The time scale on the horizontal axis is $0.1 \mathrm{sec} / \mathrm{div}$. bunch length becomes shorter when the rf voltage is driven closer to the resonance frequency.

Figure 6(b) shows the measured bunch length when we ramp the frequency of modulation down at the same time as the amplitude was ramped up. There was no observed improvement in either the adiabaticity or the minimum stable bunch width.

Figure 7 shows the distance between the SFPs and the UFPs as a function of $\frac{\nu_{m}}{\nu_{s}}$ for the conditions of our experiment. Four experimentally derived points for the distance between stable fixed points in regime (b) are shown on the upper curve.

In order to obtain narrow bunches in regime (b) we had to use a large $\epsilon$. However, in the presence of strong modulation, the approximated Hamiltonian in Eq. (6) is no longer adequate. The experimental data in Fig. 7 do not perfectly fit the prediction. We believe this is primarily because the prediction is an approximation and, as the modulation frequency gets closer to the resonance, this approximation becomes less applicable. During the experiment, measurements were also made in regime (c).

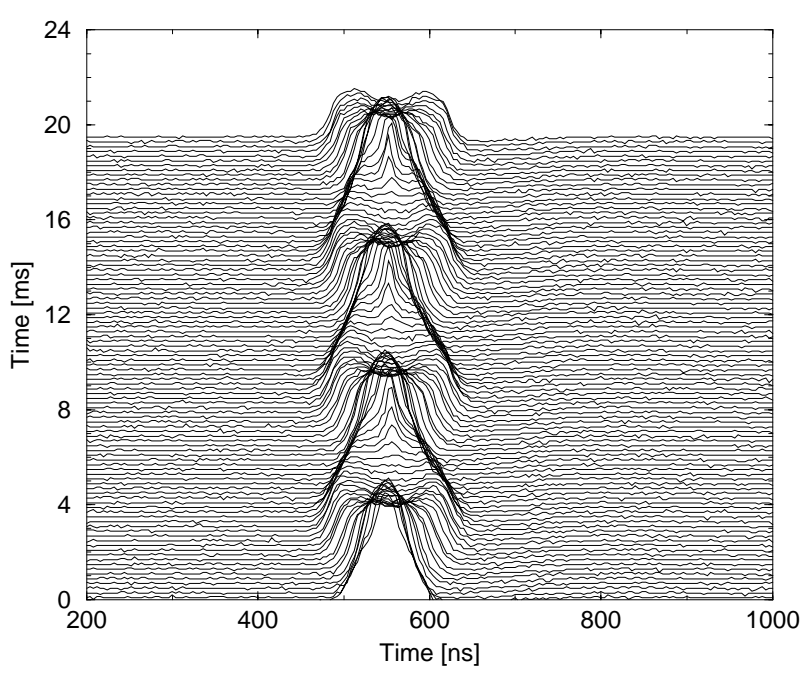

FIG. 4. Driven bunch shape oscillations seen with the wall current monitor. 

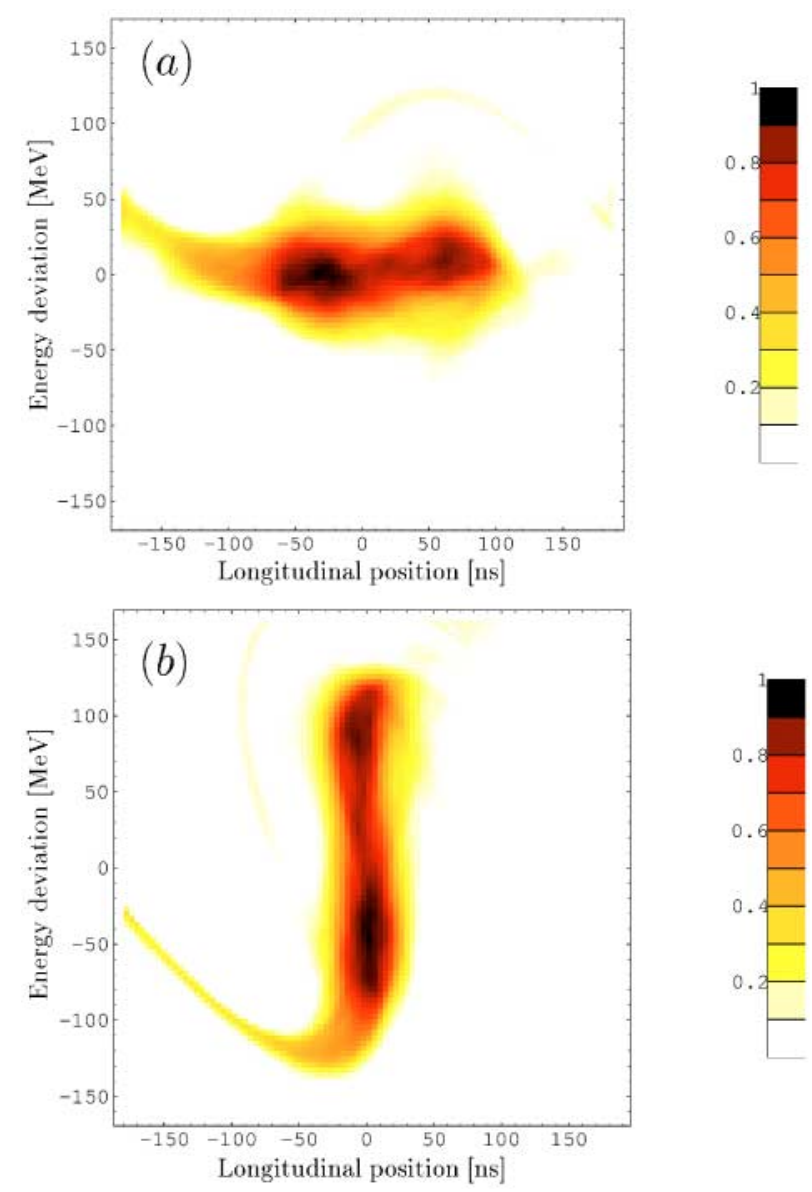

FIG. 5. (Color) Phase space reconstruction of the bunch. Part (a) shows the bunch with maximum length. In part (b), taken a quarter of a synchrotron period later, the bunch reached its minimum length.

However, we found that it is very difficult to maintain stable beam. This is also due to the strong modulation. Expanding out on the approximations of the theory may provide further insight. Alternatively, a multiparticle simulation, which uses the exact solution of the turnby-turn map [Eq. (1)], may allow us to more precisely explore this space. We have begun developing such an application, but our efforts are still in the early stages. Given existing data taken during our experiments, we expect to create simulations that match the data in all three regimes.

\section{SUMMARY}

By modulating the rf voltage at twice the synchrotron frequency, a parametric resonance can be excited. The longitudinal beam emittance can be preserved if the excitation is adiabatic. This technique allows us to obtain very short bunches. It was successfully applied in the AGS fast extraction of high intensity proton beam for the g- 2 experiment, which requires high intensity proton beams with short bunch lengths. (a)

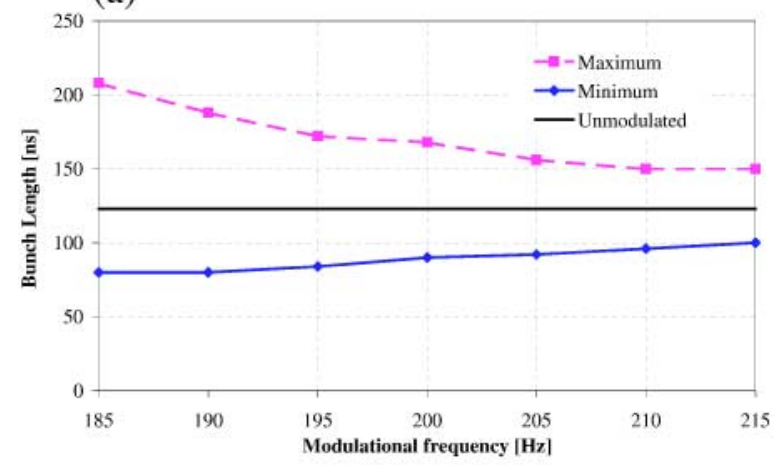

(b)

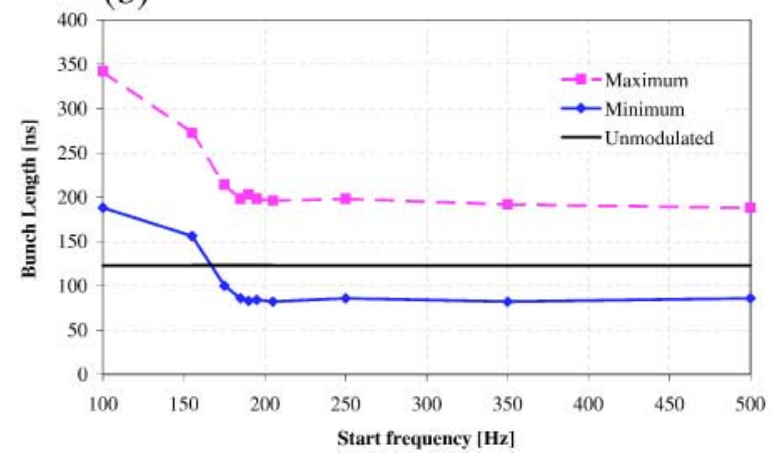

FIG. 6. (Color) (a) Measured bunch length vs modulation frequency. (b) Measured bunch length vs start frequency. The final modulation frequency is $190 \mathrm{~Hz}$ in all cases.

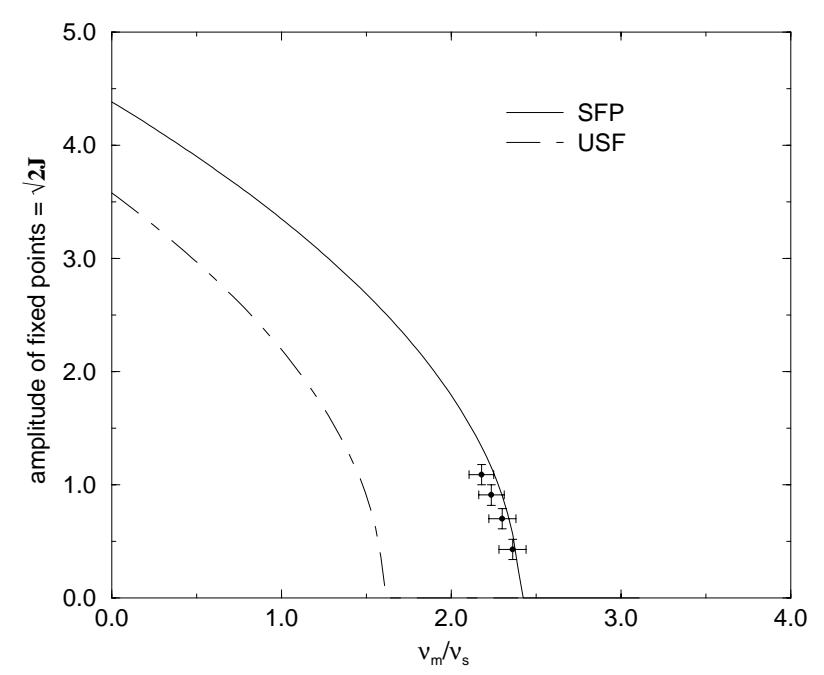

FIG. 7. Measured locations of stable fixed points.

\section{ACKNOWLEDGMENTS}

This work was supported by the U.S. Department of Energy. The authors wish to thank Steve Hancock and Mats Lindroos, CERN, for their valuable assistance in the tomographic reconstruction. We also wish to thank Kevin 
Smith, BNL, for his assistance in getting the AGS rf systems configured for our experiments.

[1] R. M. Carey et al., Phys. Rev. Lett. 82, 1632-1635 (1999).

[2] H. Huang et al., Phys. Rev. E 48, 4678 (1993).

[3] D. Li et al., Phys. Rev. E 48, 1638-1641 (1993).

[4] D. Li et al., Nucl. Instrum. Methods Phys. Res., Sect. A 364, 205-223 (1995).
[5] D. A. Edwards and M. J. Syphers, An Introduction to the Physics of High Energy Accelerators (Wiley, New York, 1993).

[6] L. D. Landau and E. M. Lifschitz, Mechanics (Pergamon, New York, 1976).

[7] R. Garoby, in Handbook of Accelerator Physics and Engineering, edited by A. Chao and M. Tigner (World Scientific, Singapore, 1999).

[8] S. Hancock, P. Knauss, and M. Lindroos, in Proceedings of the European Particle Accelerator Conference, Stockholm, Sweden, 1998 (CERN Report No. CERN-PS-98-030-RF, 1998). 